\title{
Numerical modelling of pump limiter biasing on TEXTOR-94 and Tore Supra
}

\author{
H. Gerhauser ${ }^{1}$, R. Zagórski ${ }^{2}$, H.A. Claaßen ${ }^{1}$, G. Mank ${ }^{1}$, \\ T. Loarer ${ }^{3}$, J. Gunn ${ }^{3}$ and C. Boucher ${ }^{4}$ \\ ${ }^{1}$ Institut für Plasmaphysik, Forschungszentrum Jülich GmbH, EURATOM Association, \\ Trilateral Euregio Cluster, D-52425 Jülich, Germany \\ 2 Institute of Plasma Physics and Laser Microfusion, PO Box 49, 00-908 Warsaw, Poland \\ ${ }^{3}$ Association EURATOM-CEA sur la fusion contrôlée, CEN Cadarache, F-13108 \\ St. Paul-Lez-Durance, France \\ ${ }^{4}$ Université du Québec, INRS-Énergie et Matériaux, Varennes, Canada J3X 1S2 \\ E-mail: H.Gerhauser@fz-juelich.de
}

Received 8 October 2001, accepted for publication 26 March 2002

Published 15 July 2002

Online at stacks.iop.org/NF/42/805

\begin{abstract}
The two-dimensional multifluid code TECXY has been used to model the biasing (with respect to the first wall) of the toroidal belt limiter ALT-II on the tokamak TEXTOR-94 and of the new toroidal pump limiter being installed on Tore Supra tokamak in the framework of the CIEL project. It is well known that the edge flow pattern can be influenced by the poloidal electric drifts from imposing radial electric fields. The modelling with TECXY introduces imprinted bias currents in the scrape-off layer (SOL) for the case of negative (limiter) biasing, and imprinted bias potentials for the case of positive biasing. This allowed us to simulate sufficiently well the experimental $I-V$ characteristics for either biasing of ALT-II and also reproduced the essential features and trends of the observed plasma profiles in the SOL of TEXTOR-94. For negative biasing a moderate improvement of the pumping exhaust efficiency can be achieved in the case of TEXTOR. For Tore Supra, however, only a negligible improvement of the limiter performance with biasing can be predicted, which is explained by the relatively weak drift flows in Tore Supra.
\end{abstract}

PACS numbers: 52.55.Fa, 52.40.Hf, 52.30.Ex, 52.65.Kj, 02.60.Cb, 02.60.Lj, 02.70.Bf

\section{Introduction}

The two-dimensional (2D) multifluid code TECXY [1-5] has proved to be a useful tool for investigating specific physical questions in the plasma edge of limiter tokamaks. The physical model is based on Braginskij-like equations for the background plasma and rate equations for the impurity ions. An analytical description of two groups of neutrals (cold and cx neutrals) permits taking into account plasma recycling at the limiter surface. Moreover the present version of the code incorporates drift motions and currents in a fully self-consistent way with plasma and impurity dynamics in a real curvilinear geometry of the limiter tokamak boundary layer, and the radial electric field and plasma potential in the transition layer inside the separatrix are derived from an ordinary differential equation which ensures global ambipolarity of the radial electric current.

In this paper we use a modified version of TECXY to model the experimental biasing of the toroidal belt limiter ALT-II on the tokamak TEXTOR-94 with respect to the first wall, and also the future biasing of the new toroidal pump limiter (TPL) being installed on Tore Supra tokamak in the framework of the CIEL project [6]. The experimental and theoretical investigations are motivated by the need to optimize the exhaust efficiency of the pump limiter and are carried out to explore the possibilities for improved performance of the new CIEL-TPL with biasing. It is well known that the edge flow pattern can be influenced by the poloidal electric drifts from imposing radial electric fields. Limiter biasing has been performed on several tokamaks $[7,8]$. Results on electrostatic biasing of ALT-II were reported for the first time in [9] and more recently in [8] for normal orientation of the toroidal plasma current and magnetic field and for both positive and negative biasing voltages. Meanwhile experiments were also extended to inverted orientation [10]. Modifications of floating potential, edge density and temperatures, toroidal and poloidal particle flows were measured, as well as the resulting collected and pumped particle fluxes and the particle confinement times.

The modelling with TECXY simulates sufficiently well the experimental $I-V$ characteristics for either biasing and also reproduces the essential features and trends of the observed plasma profiles in the scrape-off layer (SOL) of TEXTOR. It appears that for negative biasing the naturally existing 
perpendicular (electric and pressure gradient) drifts in the SOL can be compensated and hence limiter pumping optimized. The validated model is then applied to the analogous biasing problem in Tore Supra in order to make predictions and recommendations also for this tokamak.

\section{Physical model}

The 2D boundary layer code TECXY [1-5], like most other 2D computational plasma edge fluid models, is primarily based on the classical transport equations derived by Braginskij [11]. The model describes the electrons and various ion species in their different charge states as separate fluids. The transport along field lines is assumed to be classical and transport coefficients follow from the 21-moment Grad approximation [12]. The radial transport is assumed to be anomalous with prescribed radial transport coefficients of the order of Bohm diffusion following an Alcator-like scaling (inversely proportional to density) in the transition layer and chosen to be constant in the SOL.

There are reasons for the choice of this particular anomalous transport model. It is well confirmed by experimental results that the anomalous transport increases when approaching the plasma edge (still on closed magnetic surfaces), see e.g. [13]. Alcator-like scaling gives the increase of $D_{\perp}$ with $r$ since $n_{\mathrm{e}}$ decreases with $r$. We checked in previous papers $[1,14,15]$ also other increasing functions of $r$ in the transition layer, but the Alcator-like scaling of $D_{\perp}$ reproduces best the experimental profiles from TEXTOR $[16,3]$. The choice of constant $D_{\perp}$ and $\chi_{\perp} / n$ in the SOL is simple but gives reasonable agreement with experimental data $[2,3,5,16]$ in TEXTOR, like radial decay lengths $\lambda_{n}$ and $\lambda_{T}$. Very dedicated systematic studies on the comparison between experimentally determined transport coefficients and predictions of different models can be found in [17] for divertor tokamaks, but not for the limiter tokamaks TEXTOR and Tore Supra, where more refined transport models are not yet validated.

The dynamics of neutrals in the SOL is described by an analytical model, which accounts in a self-consistent way for recycling of deuterium ions and for sputtering and self-sputtering of impurity ions at the limiter plates.

\subsection{Multifluid equations}

For every ion species $a=i, j$ we solve the continuity, parallel momentum and energy equations. $a=i$ for deuterium ions and $a=j$ for the different charge states of impurity ions ( $j=1, \ldots, Z_{\max }, Z_{\max }$ is the impurity atomic number).

Continuity equation (particle balance)

$$
\frac{\partial n_{a}}{\partial t}+\operatorname{div}\left(n_{a} \vec{V}_{a}\right)=S_{n}^{a},
$$

where $n_{a}$ is the density of ions ( $\left.a=i, j\right)$ or electrons $(a=\mathrm{e})$, $S_{n}^{a}$ the sources (sinks) of particles and $\vec{V}_{a}$ the velocity of ions or electrons.

Equation of motion (momentum balance):

$$
\begin{gathered}
m_{a} \frac{\partial}{\partial t}\left(n_{a} \vec{V}_{a}\right)+\operatorname{div}\left(n_{a} m_{a} \vec{V}_{a} \vec{V}_{a}+p_{a} \stackrel{\leftrightarrow}{I}+\stackrel{\leftrightarrow}{\Pi}_{a}\right) \\
=n_{a} e_{a}\left(\vec{E}+\vec{V}_{a} \times \vec{B}\right)+\vec{R}_{a}+\vec{S}_{V}^{a},
\end{gathered}
$$

where $e_{a}$ is charge of particles, $m_{a}$ the mass of particles, $\vec{E}$ the electric field, $\vec{B}$ the magnetic field, $\vec{R}_{a}\left(\equiv \vec{R}_{V}^{a}+\vec{R}_{T}^{a}\right)$ the friction and thermal forces, $\vec{S}_{V}^{a}$ the sources (sinks) of momentum, $p_{a}$ the pressure, and $\stackrel{\leftrightarrow}{\Pi}_{a}$ is the viscosity tensor [18].

All ion species are assumed to have the same common temperature $T_{i}=T_{a}$, which differs from the electron temperature $T_{\mathrm{e}} \neq T_{i}$. Consequently only the following two energy equations are considered $\left(\varepsilon_{a}=\frac{1}{2} n_{a} m_{a} \vec{V}_{a}^{2}\right)$ :

$$
\begin{aligned}
& \frac{3}{2} \frac{\partial n_{\mathrm{e}} T_{\mathrm{e}}}{\partial t}+\nabla \cdot\left(\frac{5}{2} n_{\mathrm{e}} \vec{V}_{\mathrm{e}} T_{\mathrm{e}}+\vec{q}_{\mathrm{e}}\right) \\
& =-e n_{\mathrm{e}} \vec{V}_{\mathrm{e}} \cdot \vec{E}+\vec{R}_{\mathrm{e}} \cdot \vec{V}_{\mathrm{e}}+Q_{\mathrm{e}}+S_{E}^{\mathrm{e}} \\
& \frac{\partial}{\partial t} \sum_{a}\left(\frac{3}{2} n_{a} T_{i}+\varepsilon_{a}\right) \\
& \quad+\nabla \cdot \sum_{a}\left(\frac{5}{2} n_{a} \vec{V}_{a} T_{i}+\vec{V}_{a} \varepsilon_{a}+\vec{q}_{a}+\vec{V}_{a} \cdot \stackrel{\leftrightarrow}{\Pi}_{a}\right) \\
& \quad=\vec{j} \cdot \vec{E}+e n_{\mathrm{e}} \vec{V}_{\mathrm{e}} \cdot \vec{E}-\vec{R}_{\mathrm{e}} \cdot \vec{V}_{\mathrm{e}}-Q_{\mathrm{e}}+\sum_{a} S_{E}^{a}
\end{aligned}
$$

The plasma current is $\vec{j}=\sum_{a} e_{a} n_{a} \vec{V}_{a}-e n_{\mathrm{e}} \vec{V}_{\mathrm{e}}$ and all other symbols have their usual meaning $[1,3,11,14]$. The energy source terms contain both the work of the electric field (Joule heating) and the collisional energy exchange between electrons and ions $\left(Q_{\mathrm{e}}\right)[14]$

\subsection{Drifts and currents}

The geometry of the boundary layer is sketched in figure 1 for normal polarity (orientation) of the plasma current $I_{\mathrm{p}}$ and the main (toroidal) magnetic field $B_{\phi}$ in case of TEXTOR-94 tokamak. The coordinates $x, y$ and $z$ correspond to the poloidal, radial and toroidal directions, respectively. The parallel $(\|)$ direction is along the total magnetic field, the perpendicular $(\perp)$ direction is restricted to be within the magnetic flux surface, such that the $(\|, \perp)$-axes are rotated against the $(z, x)$-axes by a few degrees. For inverted orientation of $I_{\mathrm{p}}$ and $B_{\phi}$ both $\|$ and $\perp$ must be inverted. The

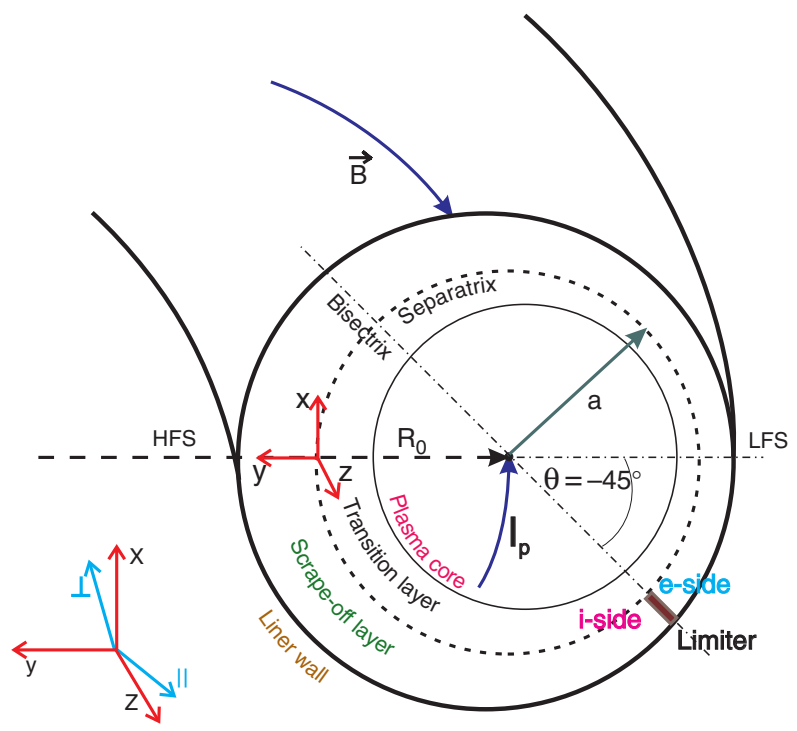

Figure 1. Boundary layer geometry for TEXTOR tokamak toroidal belt limiter. 
abbreviation HFS means of course high field side, LFS is the low field side, i-side is the ion side of the limiter, and e-side is the electron side (because if we consider the parallel component of the main plasma current, then the ions would hit the i-side and the electrons the e-side). The scrape-off layer is often abbreviated as SOL and the separatrix as LCMS (last closed magnetic flux surface). The width of the transition layer between separatrix and plasma core is a few $\mathrm{cm}$ like the width of the SOL.

In order to consider drift motions and currents in the tokamak boundary layer additional equations have to be added, which were obtained from the radial and perpendicular components of the equations of motion for electrons and ions. We have perpendicular drift velocities and, in addition to the radial diffusion velocities $-D_{y}^{a} \partial \ln n_{a} / h_{y} \partial y$, also radial drift velocities:

$$
\begin{aligned}
& V_{\mathrm{e} \perp}=\frac{1}{B h_{y}}\left[\frac{1}{e n_{\mathrm{e}}} \frac{\partial p_{\mathrm{e}}}{\partial y}-\frac{\partial \Phi}{\partial y}\right] \\
& V_{a \perp}=\frac{1}{e_{a} n_{a} B} \frac{1}{h_{y}}\left[-\frac{\partial}{\partial y}\left(p_{a}-b_{a}\right)-e_{a} n_{a} \frac{\partial \Phi}{\partial y}\right. \\
& \left.+\left(3 b_{a}+m_{a} n_{a} V_{a \|}^{2}\right) \frac{\partial}{\partial y} \ln \left(h_{z} b_{z}\right)\right], \\
& V_{\mathrm{e} y}^{\mathrm{d}}=\frac{-b_{z}}{B h_{x}}\left(\frac{1}{e n_{\mathrm{e}}} \frac{\partial p_{\mathrm{e}}}{\partial x}-\frac{\partial \Phi}{\partial x}\right), \\
& V_{a y}^{\mathrm{d}}=\frac{1}{e_{a} n_{a} B}\left[\frac{B}{h_{x} h_{z}} \frac{\partial}{\partial x}\left(\frac{h_{z} b_{z}}{B}\left(p_{a}-b_{a}\right)\right)+e_{a} n_{a} \frac{b_{z}}{h_{x}} \frac{\partial \Phi}{\partial x}\right. \\
& -\left(2 p_{a}+b_{a}+m_{a} n_{a} V_{a \|}^{2}\right) \frac{b_{z}}{h_{x}} \frac{\partial}{\partial x} \ln \left(h_{z} b_{z}\right) \\
& +m_{a} n_{a} \vec{V}_{a} \cdot \nabla V_{a \perp}-\left(S_{V \perp}^{a}-m_{a} V_{a \perp} S_{n}^{a}\right) \\
& \left.-\frac{1}{\sqrt{g}} \frac{\partial}{\partial y} \frac{\sqrt{g}}{h_{y}^{2}} \eta_{\perp}^{a} \frac{\partial V_{a \perp}}{\partial y}\right] \text {. }
\end{aligned}
$$

Here $\Phi$ is the plasma potential, $b_{a} \equiv 0.5 \Pi_{|\||}^{a}$, and $\eta_{\perp}$ is the perpendicular shear viscosity. Note that $\sqrt{g}=h_{x} h_{y} h_{z}=$ $h_{\perp} h_{y} h_{\|} ; h_{x}, h_{y}$ and $h_{z}$ as well as $h_{\perp}$ and $h_{\|}$are the metric coefficients, whereas $b_{x}=-B_{\theta} / B$ and $b_{z}=B_{\phi} / B$ are ratios between poloidal, respectively, toroidal and total magnetic field. Further details of the geometry and metric coefficients are given in [18].

The radial and perpendicular components of the plasma current $\left(j_{y}, j_{\perp}\right)$ are derived from equations (5)-(8):

$$
\begin{aligned}
j_{y}= & \frac{b_{z}}{B h_{x}}\left[\frac{B}{b_{z} h_{z}} \frac{\partial}{\partial x}\left(\frac{h_{z} b_{z}}{B}\left(p-\sum_{a} b_{a}\right)\right)\right. \\
& \left.-\left(2 p+\sum_{a} b_{a}+\sum_{a} m_{a} n_{a} V_{a \|}^{2}\right) \frac{\partial}{\partial x} \ln \left(h_{z} b_{z}\right)\right] \\
& +\frac{1}{B}\left[\sum_{a} m_{a} n_{a} \vec{V}_{a} \cdot \nabla V_{a \perp}-\sum_{a}\left(S_{V_{\perp}}^{a}-m_{a} V_{a \perp} S_{n}^{a}\right)\right. \\
& \left.-\sum_{a} \frac{1}{\sqrt{g}} \frac{\partial}{\partial y} \frac{\sqrt{g}}{h_{y}^{2}} \eta_{\perp}^{a} \frac{\partial V_{a \perp}}{\partial y}\right],
\end{aligned}
$$

$$
\begin{aligned}
j_{\perp}= & -\frac{1}{B}\left[\frac{\partial}{\partial y}\left(p-\sum_{a} b_{a}\right)\right. \\
& \left.-\sum_{a}\left(3 b_{a}+m_{a} n_{a} V_{a \|}^{2}\right) \frac{1}{h_{y}} \frac{\partial}{\partial y}\left(h_{z} b_{z}\right)\right],
\end{aligned}
$$

where $p\left(\equiv p_{\mathrm{e}}+\sum_{a} p_{a}\right)$ is the total pressure. The last term in the radial drift velocity $V_{a y}^{\mathrm{d}}$ and the radial current $j_{y}$ is related to the force from the perpendicular shear viscosity $\eta_{\perp}^{a}$. It may be of the same order as the competing forces, namely, the Lorentz force, the $\nabla B$ and centrifugal forces and the forces from inertia and momentum transfer from neutrals. The inclusion of this perpendicular shear in the force balance and the global ambipolarity constraint for the radial currents allows us to determine unambiguously the perpendicular electric drift and hence the radial electric field in the transition layer. It was shown in [18] that the mathematical problem may be reduced to the solution of a third order ordinary differential equation for the radial dependence of the plasma potential (second order for the radial electric field).

The poloidal component of the current can be expressed by the parallel and perpendicular components: $j_{x}=b_{x} j_{\|}+b_{z} j_{\perp}$. Using Kirchhoff's law,

$$
\operatorname{div} \vec{j}=\frac{1}{\sqrt{g}}\left(\frac{\partial}{\partial x} \frac{\sqrt{g}}{h_{x}} j_{x}+\frac{\partial}{\partial y} \frac{\sqrt{g}}{h_{y}} j_{y}\right)=0,
$$

we can introduce the stream function $\Psi(x, y)$ by

$$
\frac{\sqrt{g}}{h_{x}} j_{x}=-\frac{\partial \Psi}{\partial y}, \quad \frac{\sqrt{g}}{h_{y}} j_{y}=\frac{\partial \Psi}{\partial x} .
$$

It is determined up to some function of $y$ :

$\Psi(x, y)=\tilde{\Psi}(x, y)+F(y)=\int_{x_{\mathrm{p}}} \frac{\sqrt{g}}{h_{y}} j_{y} \mathrm{~d} x+F(y)$.

Hence the poloidal and parallel components of the current are:

$$
\begin{gathered}
j_{x}=\tilde{j}_{x}+j_{x \mathrm{c}} \equiv-\frac{h_{x}}{\sqrt{g}} \frac{\partial \tilde{\Psi}}{\partial y}-\frac{h_{x}}{\sqrt{g}} \frac{\mathrm{d} F}{\mathrm{~d} y}, \\
j_{\|}=\tilde{j}_{\|}+j_{\| \mathrm{c}}, \quad \tilde{j}_{\|}=-\frac{h_{x}}{b_{x} \sqrt{g}} \frac{\partial \tilde{\Psi}}{\partial y}-\frac{b_{z}}{b_{x}} j_{\perp}, \\
j_{\| \mathrm{c}}=-\frac{h_{x}}{b_{x} \sqrt{g}} \frac{\mathrm{d} F}{\mathrm{~d} y} .
\end{gathered}
$$

$F$ can now be determined from the condition for a unique electrostatic potential by using Ohm's law along the magnetic field:

$\Delta \Phi_{\mathrm{es}}-\Delta \Phi_{\mathrm{is}}=\int_{x_{\mathrm{is}}}^{x_{\mathrm{es}}}\left(\frac{1}{e n_{\mathrm{e}}} \frac{\partial p_{\mathrm{e}}}{\partial x}+\frac{\alpha_{T}}{e} \frac{\partial T_{\mathrm{e}}}{\partial x}-\frac{h_{x}}{b_{x}} \frac{j_{\|}}{\sigma_{\|}}\right) \mathrm{d} x$.

Thus

$$
\begin{aligned}
F^{\prime} & \equiv \frac{\mathrm{d} F}{\mathrm{~d} y} \\
& =\frac{\left(\Delta \Phi_{\mathrm{es}}-\Phi_{0 \mathrm{es}}\right)-\left(\Delta \Phi_{\mathrm{is}}-\Phi_{0 \mathrm{is}}\right)+\int_{x_{\mathrm{is}}}^{x_{\mathrm{es}}}\left(h_{x} / b_{x}\right)\left(\tilde{j}_{\|} / \sigma_{\|}\right) \mathrm{d} x}{\int_{x_{\mathrm{is}}}^{x_{\mathrm{es}}}\left(h_{x}^{2} / b_{x}^{2}\right)(1 / \sqrt{g}) \mathrm{d} x} .
\end{aligned}
$$


The potential drop in the Langmuir sheath $\Delta \Phi \geqslant 0$ is given by $\Delta \Phi=\Delta \Phi^{\mathrm{sh}}-T_{\mathrm{e}} / e \ln \left(1-j_{x} / \sum_{a} e_{a} n_{a} V_{a x}\right)$ where $\Delta \Phi^{\mathrm{sh}}\left(\sim 3 T_{\mathrm{e}} / e\right)$ is the drop without poloidal current. Since $j_{x}=\tilde{j}_{x}-h_{x} F^{\prime} / \sqrt{g}$ the equation for $F^{\prime}$ is strongly nonlinear in the SOL, but there the function $F^{\prime}$ uniquely determines potential and currents. However, in the transition layer, where $\Delta \Phi=0$, the potential at one line (e.g. bisectrix, $x=x_{\text {bis }}$ ) has to be given in order to close the system of equations. It can be found from the above mentioned differential equation for $E_{r}=-\left(1 / h_{y}\right) \partial \Phi / \partial y$ obtained from the global ambipolarity constraint [18], which on closed magnetic surfaces means: $\oint_{x} j_{y} h_{z} h_{x} \mathrm{~d} x=0$, or

$$
\begin{aligned}
0= & -\oint \frac{h_{z} h_{x}}{B}\left[\frac{b_{z}}{h_{x}}\left(2 p+\sum_{a}\left(b_{a}+m_{a} n_{a} V_{a \|}^{2}\right)\right) \frac{\partial}{\partial x} \ln \left(h_{z} b_{z}\right)\right. \\
& \left.+\sum_{a} m_{a} c_{\mathrm{s}}^{a} b_{x} P_{2}^{a}\right] \mathrm{d} x+\oint \frac{h_{z} h_{x}}{B}\left[\sum_{a} m_{a} V_{a \perp}\left(P_{1}^{a}+S_{n}^{a}\right)\right. \\
& \left.+\sum_{a} m_{a} n_{a}\left(V_{a x} \frac{1}{h_{x}} \frac{\partial}{\partial x}+V_{a y} \frac{1}{h_{y}} \frac{\partial}{\partial y}\right) V_{a \perp}\right] \mathrm{d} x \\
& -\oint \frac{h_{z} h_{x}}{B} \frac{1}{\sqrt{g}} \frac{\partial}{\partial y}\left(\frac{\sqrt{g}}{h_{y}^{2}} \sum_{a} \eta_{\perp}^{a} \frac{\partial V_{a \perp}}{\partial y}\right) \mathrm{d} x,
\end{aligned}
$$

depending linearly on $V_{a \perp}$. The source term from the momentum transfer by cx with neutrals has been written as: $S_{V \perp}^{a} \equiv-m_{a} V_{a \perp} P_{1}^{a}+m_{a} c_{\mathrm{s}}^{a} b_{x} P_{2}^{a}$, where $c_{\mathrm{s}}^{a}$ is the sound speed, and $P_{1}^{a}, P_{2}^{a}$ are given in subsection 2.4.

Let us denote $\Phi=\Phi^{*}+\tilde{\Phi}$, where $\Phi^{*}=\Phi^{*}(y)=$ $\Phi\left(x_{\text {bis }}, y\right)$ and $\tilde{\Phi}=\tilde{\Phi}(x, y)$ with $\tilde{\Phi}\left(x_{\text {bis }}, y\right) \equiv 0$. The condition for a unique electrostatic potential $\oint \mathrm{d} \Phi=$ $\oint \partial \Phi / \partial x \mathrm{~d} x=\oint \partial \tilde{\Phi} / \partial x \mathrm{~d} x=0$ means that $\tilde{\Phi}(x, y)$ can be found for every $y$ by integrating the parallel Ohm's law along $x$. At the separatrix $\Phi^{*}$ and $\mathrm{d} \Phi^{*} / \mathrm{d} y$ are given by the values obtained in the SOL. At the core boundary we use the condition, that the total poloidally circulating ion current from perpendicular ion motion vanishes: $\left.\oint \sqrt{g} b_{z} j_{i \perp} \mathrm{d} x\right|_{y=0}=$ $\left.\oint \sqrt{g} b_{z} \sum_{a} e_{a} n_{a} V_{a \perp} \mathrm{d} x\right|_{y=0}=0$, which yields $\mathrm{d} \Phi^{*} /\left.\mathrm{d} y\right|_{y=0}$.

Considering all quantities including $V_{a y}, V_{a x}, V_{a \|}$ as given and writing the expression for $V_{a \perp}$ in terms of the unknown radial electric field, the ambipolarity constraint (equation (18)) is finally transformed into a linear differential equation of third order for $\Phi^{*}$ :

$$
B(y) \frac{\mathrm{d} \Phi^{*}}{\mathrm{~d} y}+C(y) \frac{\mathrm{d}^{2} \Phi^{*}}{\mathrm{~d} y^{2}}+D(y) \frac{\mathrm{d}^{3} \Phi^{*}}{\mathrm{~d} y^{3}}=A(y)
$$

where $A(y), B(y), C(y), D(y)$, are known functions of plasma parameters [18]. The numerical solution of the differential equation is incorporated into the iteration loop for determining the drift quantities.

We note that in the above formulation equation (18) Kirchhoff's law is exploited for the non-turbulent (timeaveraged) values of the current density, the anomalous part of which being assumed to disappear due to ambipolarity in context with a common anomalous diffusion coefficient $D_{y}^{a}=D_{y}^{\mathrm{e}}$ for all charge carriers. There exist, of course, parallel and cross-field current density fluctuations, which by themselves satisfy Kirchhoff's law under quasineutrality conditions and play a decisive role when estimating the diffusion $D_{y}$ at the plasma edge from $\gamma / k_{\perp}^{2}$, where $\gamma$ is the linear growth rate of ideal or resistive ballooning or interchange modes at the most unstable wave number $k_{\perp}$.

\subsection{Boundary conditions}

The boundary conditions fixing densities, temperatures and flow velocities (or their gradients) of the charge carriers at the boundaries of the integration domain, which (in the case of TEXTOR) extends poloidally from one limiter side $\left(x_{i}=-1.45 \mathrm{~m}\right)$ to the other limiter side $\left(x_{\mathrm{e}}=1.45 \mathrm{~m}\right)$ and radially from $r=42 \mathrm{~cm}$ to $r=50 \mathrm{~cm}$ with the separatrix at $a=46 \mathrm{~cm}$, are indicated in figure 2 . At the core boundary a total power input flux and a total particle input flux are specified. At the wall we have used decay lengths as boundary conditions. At the auxiliary boundary, which continues the limiter sides into the transition layer, we have assumed that all quantities are continuous and poloidally periodic. At the limiter we adopt the conditions $q_{\mathrm{e} x}=\delta_{\mathrm{e}} n_{\mathrm{e}} V_{\mathrm{e} x} T_{\mathrm{e}}$, $q_{a x}=\delta_{a} n_{a} V_{a x} T_{i}$ for the energy equations and $V_{a x}=b_{x} V_{a \|}+$ $b_{z} V_{a \perp}= \pm b_{x} c_{\mathrm{s}}^{a}$ for the momentum balance equations. Here the perpendicular drift velocity $V_{a \perp}$ is according to equation (6) composed of an electric drift $V_{a \perp}^{\mathrm{E}}$ which involves $\partial \Phi / \partial y$ and a diamagnetic drift $V_{a \perp}^{\mathrm{D}}$ which is dominated by the term with $\partial p_{a} / \partial y$. The latter boundary condition is certainly a contentious issue and corresponds to the 'intuitive' boundary condition mentioned in [19]. It replaces a more complicated form derived in [20] from MHD equations at the entrance of the magnetic presheath, which contains drifts driven by temperature and density gradients appearing as a consequence of inertia terms in the momentum balance. With the present notation and $\gamma_{\mathrm{e}}=\gamma_{i}=1$ it reads

$$
\begin{gathered}
{\left[b_{x} V_{a \|}+b_{z} V_{a \perp}^{\mathrm{E}}+b_{z} V_{a \perp}^{\mathrm{D}}\right]\left[b_{x} V_{a \|}+b_{z} V_{a \perp}^{\mathrm{E}}+b_{z} \frac{k T_{\mathrm{e}}}{e n_{\mathrm{e}} B} \frac{\partial n_{\mathrm{e}}}{h_{y} \partial y}\right]} \\
=b_{x}^{2}\left(c_{\mathrm{s}}^{a}\right)^{2}+b_{x} b_{z} \frac{k T_{\mathrm{e}}+k T_{a}}{e_{a} B} \frac{\partial V_{a \|}}{h_{y} \partial y}
\end{gathered}
$$

The structure of the velocity boundary condition advocated in equation (73) of [19] is very similar, but our term with $V_{a \perp}^{\mathrm{D}}$ in the first square bracket of equation (20) and our term with $T_{a} \partial V_{a \|} / \partial y$ on the right-hand side are missing. We think however that the ion diamagnetic drift cannot be neglected at the magnetic presheath entrance, and it was shown in [20] by a kinetic derivation that a similar structure as above is valid deeply inside the magnetic presheath at the transition to the Debye sheath, where the ion thermal motion is effectively cooled away and electric drifts dominate the ion motion, such that only there $V_{a \perp}^{\mathrm{E}}+V_{a \perp}^{\mathrm{D}}$ can and must be substituted by $V_{a \perp}^{\mathrm{E}}$. We admit that by using the simplified intuitive boundary condition, which retains only the first square bracket of the

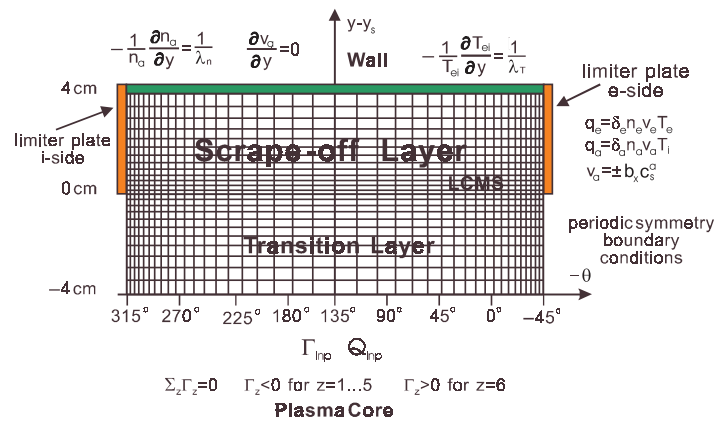

Figure 2. Numerical mesh and boundary conditions (TEXTOR). 
above more complicated condition equation (20), the effect of diamagnetic drift flows may be overestimated. But the effect of the electric drift is according to [21] described correctly, and it is usually larger than the correction by the diamagnetic drifts, especially in the present context of investigating large electric drifts from applying large bias voltages. So we leave the use of more refined boundary conditions to the future when the controversy about the correct structure will be settled.

\subsection{Neutral model}

The dynamics of deuterium in the neighbourhood of the belt limiter is described by an analytical model, which accounts in a self-consistent way for recycling of plasma ions at the limiter plates [3]. Two groups of neutrals are considered: fast (hot) and slow (cold) neutrals $\left(N_{\mathrm{D}}=N_{\mathrm{D}}^{\mathrm{f}}+N_{\mathrm{D}}^{\mathrm{s}}\right)$. The profile of deuterium atoms close to each side of the limiter is prescribed by a product of exponential functions (plate $=\mathrm{e}$-side or plate $=\mathrm{i}$-side):

$$
\begin{gathered}
N_{\mathrm{D}}^{\mathrm{f}, \mathrm{s}}(x, y)=N_{\text {plate }}^{\mathrm{f}, \mathrm{s}} \exp \left(-\left|\int_{x_{\text {plate }}}^{x} \frac{\mathrm{d} x}{\lambda_{x}^{\mathrm{f}, \mathrm{s}}}\right|\right) \\
\quad \times \exp \left(-\frac{\left(y_{\mathrm{M}}(x)-y\right)^{2}}{\left(\lambda_{y}^{\mathrm{f}, \mathrm{s}}\right)^{2}}\right),
\end{gathered}
$$

where $\lambda_{x, y}^{\mathrm{f}}=\left|v_{\mathrm{D} x, y}^{\mathrm{f}}\right| /\left(n_{\mathrm{e}} \sqrt{\alpha_{i}^{\mathrm{D}} \alpha_{\mathrm{cx}}^{\mathrm{D}}}\right), \lambda_{x, y}^{\mathrm{s}}=\left|v_{\mathrm{D} x, y}\right|^{\mathrm{s}} /\left(n_{\mathrm{e}} \alpha_{i}^{\mathrm{D}}\right)$, $\alpha_{i}^{\mathrm{D}}, \alpha_{\mathrm{cx}}^{\mathrm{D}}$ are the rate coefficients for ionization and charge exchange processes, respectively, $x_{\text {plate }}$ is the position of the limiter plate, while $y_{M}\left(x_{\text {plate }}\right)$ is the position of the maximum neutral particle reflux from the target plate.

The deuterium neutral densities $N_{\text {plate }}^{\mathrm{f}}=\frac{3}{4} N_{\text {plate }}$ and $N_{\text {plate }}^{\mathrm{s}}=\frac{1}{4} N_{\text {plate }}$ at the limiter plate depend on the recycling coefficient $R$ and can be found from the integral relation:

$$
\begin{gathered}
\int_{\mathrm{VOL}} N_{\mathrm{D}}(x, y) \alpha_{i}^{\mathrm{D}}(x, y) n_{\mathrm{e}}(x, y) \mathrm{d} V \\
=R \int_{\text {plate }}\left|n_{i} v_{x}^{i}\right|\left(x_{\text {plate }}, y\right) \mathrm{d} S,
\end{gathered}
$$

where VOL is the volume of the SOL and transition layer. The recycling coefficient for deuterium $R$ is an external parameter in our model and $R=0.75$ has been assumed in all of the calculations, meaning that $75 \%$ of the deuteron reflux to the limiter arises from ionization of neutrals in the considered boundary layer and $25 \%$ from the influx across the interface with the plasma core. We have tested also other values of the recycling parameter $[16,3]$, but it comes out from the comparison with experimental results that $R=0.75$ seems to be a good choice for present-day high density TEXTOR discharges $[16,2,3,5,14]$. It has also been checked that there is very weak influence of the recycling coefficient $(0.7<R<0.8)$ on the results with biasing.

It is assumed that the directed velocity of the (slow and fast) neutrals is equal to the thermal velocity with the corresponding temperature, and that it points almost into the poloidal direction (away from the limiter). For the fast (hot) neutrals the deuterium temperature $T_{\mathrm{D}}^{\mathrm{f}}$ is determined in the model as an average of two equilibrium temperatures: $T_{\mathrm{D}}^{\mathrm{f}}=0.5\left(T_{\mathrm{D}}^{\mathrm{cx}}+T_{\mathrm{D}}^{\mathrm{eq}}\right)$. The temperature $T_{\mathrm{D}}^{\mathrm{cx}}$ follows from the assumption of energy equilibrium between inelastic collision processes:

$$
\begin{gathered}
\alpha_{i}^{\mathrm{D}} E_{\mathrm{D}}^{\mathrm{cx}}+\alpha_{\mathrm{cx}}^{\mathrm{D}}\left(E_{\mathrm{D}}^{\mathrm{cx}}-E_{i}\right)=0, \\
E_{\mathrm{D}}^{\mathrm{cx}}=1.5 T_{\mathrm{D}}^{\mathrm{cx}}+\frac{m_{i}}{2}\left(v_{\mathrm{D}}^{\mathrm{f}}\right)^{2}, \quad E_{i}=1.5 T_{i}+\frac{m_{i}}{2} v_{i, \text { tot }}^{2} \\
v_{i, \text { tot }}^{2}=\left(v_{i \|}\right)^{2}+\left(v_{i \perp}\right)^{2}
\end{gathered}
$$

whereas the temperature $T_{\mathrm{D}}^{\mathrm{eq}}$ is determined by the condition:

$$
1.5 T_{\mathrm{D}}^{\mathrm{eq}}+\frac{m_{i}}{2}\left(v_{\mathrm{D}}^{\mathrm{f}}\right)^{2}=E_{i}=1.5 T_{i}+\frac{m_{i}}{2} v_{i, \mathrm{tot}}^{2}
$$

For the slow (cold) neutrals it is assumed that they are born with the constant temperature $T_{\mathrm{D}}^{\mathrm{s}}=5 \mathrm{eV}$. This value overestimates typical Franck-Condon energies of $2-3 \mathrm{eV}$, but it was used in all our calculations $[16,2,3,5,14]$ aiming at detailed comparison of our model with experimental measurements, and the influence of this model parameter on the results of calculations was found to be very weak. $T_{\mathrm{D}}^{\mathrm{s}}$ mainly determines the ionization e-folding length along field lines which is proportional to $\sqrt{T}$. Additionally only $25 \%$ of all neutrals are considered as cold.

The source term in equation (18) can be written as

$$
\begin{gathered}
P_{1}^{i}=\sum_{\mathrm{f}, \mathrm{s}} N_{\mathrm{D}}^{\mathrm{f}, \mathrm{s}} \alpha_{\mathrm{cx}}^{\mathrm{D}}+n_{i} \beta_{\mathrm{rec}}^{\mathrm{D}}, \\
c_{\mathrm{s}}^{i} b_{x} P_{2}^{i}=\sum_{\mathrm{f}, \mathrm{s}} N_{\mathrm{D}}^{\mathrm{f}, \mathrm{s}} v_{\mathrm{D} \perp}^{\mathrm{f}, \mathrm{s}}\left(\alpha_{i}^{\mathrm{D}}+\alpha_{\mathrm{cx}}^{\mathrm{D}}\right),
\end{gathered}
$$

where $\beta_{\text {rec }}^{\mathrm{D}}$ is the recombination rate coefficient. We do not describe the impurity neutral model here, because for the biasing calculations the code was run for pure deuterium plasma only.

The simplified model for neutrals seems to work quite well. The comparison with experimental data $[16,2,3,5,14]$ is reasonable, the sensitivity on the free model parameters is small, and we have compared the model also with more complete B2-EIRENE calculations in the case of modelling of MARFE phenomena [2,5] where the agreement was quite satisfactory.

\subsection{Modelling of biasing}

We want to bias the limiter against the liner wall. But since the wall potential (and the sheath potential in front of the wall) does not enter our physical model, it is impossible to impose directly the bias potential by boundary conditions in our calculations. The reason is such that we assumed the magnetic field lines to be parallel to the liner wall. Consequently at the wall we can only specify plasma parameters like $T_{\mathrm{e}}, T_{i}, n_{i}$, $v_{i}$ (or their derivatives) but not the plasma potential. There is no valid plasma sheath theory for a surface-parallel magnetic field in front of a wall. Thus, in order to simulate the biasing it was necessary, in the case of negative limiter biasing, to imprint a bias current in the SOL from outside by prescribing a divergence-free $\vec{j}_{\text {bias }}$, and to assume a prescribed bias potential profile in the case of positive biassing. This subdivision was made in order to better reproduce the experimentally found $I-V$ characteristics (section 3 ). The imprinted bias 
current was introduced with the help of an additional stream function $\Psi_{\text {bias }}$ :

$\Psi_{\text {bias }}= \begin{cases}\frac{1}{2} \Delta \Psi_{\text {bias }}\left(2 \frac{x-x_{\text {bis }}}{x_{\mathrm{e}}-x_{i}}\right)^{\beta}\left(\frac{y-y_{\mathrm{s}}}{y_{\mathrm{w}}-y_{\mathrm{s}}}\right)^{\kappa} & \text { for } y \geqslant y_{\mathrm{s}}, \\ 0 & \text { for } y<y_{\mathrm{s}},\end{cases}$

where $y_{\mathrm{w}}$ is the wall position and $y_{\mathrm{s}}$ is the position of the separatrix. $x_{i}, x_{\mathrm{e}}$ are the poloidal positions of the limiter ion and electron sides, respectively, and $x_{\mathrm{bis}}$ is the position of the bisectrix. For $\beta=\kappa=3$ we achieve a parabolic increase of currents from the separatrix towards the corner points (where the limiter is insulated against the wall). But we checked that the results depend weakly on the chosen $\beta, \kappa$ values. The stream lines give the bias current density $\vec{j}_{\text {bias }}$ and are shown schematically in figure 3 .

$$
\frac{\sqrt{g}}{h_{x}} j_{x \text { bias }}=-\frac{\partial \Psi_{\text {bias }}}{\partial y}, \quad \frac{\sqrt{g}}{h_{y}} j_{y \text { bias }}=\frac{\partial \Psi_{\text {bias }}}{\partial x}
$$

$\Delta \Psi_{\text {bias }}$ is defined by the total (negative) bias current to the wall $I_{y \text { bias }}$ :

$$
\Delta \Psi_{\text {bias }}=\frac{I_{y \text { bias }}}{2 \pi R_{0}}<0
$$

where $R_{0}$ is the major radius of the device. In the case of positive biasing the potential in the SOL $\left(y \geqslant y_{\mathrm{s}}\right)$ was modified by an imprinted bias potential which is parabolically increasing from the separatrix to the wall:

$$
\begin{aligned}
& \Phi(x, y)=\Phi_{0}(x, y)-\Phi_{\text {bias }} \\
& =\Phi_{0}(x, y)-\Delta \Phi_{\text {bias }}\left(\frac{y-y_{\mathrm{s}}}{y_{\mathrm{w}}-y_{\mathrm{s}}}\right)^{2}
\end{aligned}
$$

where $\Phi_{0}$ was the potential calculated in the SOL without biasing (which is again slightly modified as a result of the arising bias currents). Note that in case of negative biasing the electric current to the wall is carried mainly by the electrons and in case of positive biasing by the ions, i.e. by those charge carriers which are repelled from the limiter.

Of course, we have checked the influence of the shape of the additional stream function $\Psi_{\text {bias }}$ on the results of calculations by taking different powers $\kappa$ in equation (27). It appears however that the results of simulations depend very weakly on this parameter. The same is true also for the shape of the imprinted bias potential equation (30).

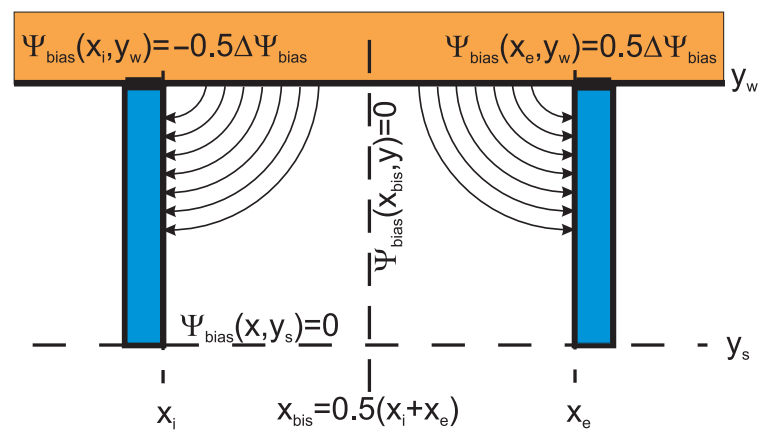

Figure 3. Schematics of the additional bias current.

\section{Results of calculations for TEXTOR and comparison with experiment}

We performed calculations with the TECXY code for a high density auxiliary heated TEXTOR-94 discharge in deuterium but neglected the effect of impurities. The belt limiter ALT-II is at $\theta=-45^{\circ}$ position, the total magnetic field $B=2.25 \mathrm{~T}$, the plasma current $I_{\mathrm{p}}=-350 \mathrm{kA}$ and the Shafranov shift $\Delta=6 \mathrm{~cm}$. The anomalous transport in radial direction is determined by the coefficients $D_{y}=1.5 \mathrm{~m}^{2} \mathrm{~s}^{-1}$, $\eta_{y}=\frac{1}{3} m_{i} n_{i} D_{y}$ and $\chi_{y}^{\mathrm{e}} / n_{\mathrm{e}}=\frac{3}{2} \chi_{y}^{i} / n_{i}=2 D_{y}$. For the present case the input particle flux to the SOL was $\Gamma_{\text {inp }}=5.5 \times 10^{21} \mathrm{~s}^{-1}$ and the power input $Q_{\text {inp }}=1 \mathrm{MW}$.

Our calculations have been compared with experimental results from TEXTOR-94. In the experiment the ALT-II belt limiter was biassed negatively or positively with respect to the liner and to the bumper limiter. We used a Mach or Gundestrup probe [8] located in the top position to measure the plasma flow. A reasonable Mach number can only be measured for radii smaller than $r=49 \mathrm{~cm}$, because of obstacles such as antenna limiter and poloidal limiter. Contrary to this the rake probe and the thermal lithium and helium beams, which are mounted in the equatorial plane $90^{\circ}$ clockwise separated from the Gundestrup probe, measure for all radii the influence of the applied electric fields. At their flux lines there are no obstacles between the bumper limiter and the ALT-II limiter.

The resulting measured $I-V$ characteristics versus the calculated ones are shown in figure 4 for both normal as well as inverted $\left(B_{\phi} \rightarrow-B_{\phi}, I_{\mathrm{p}} \rightarrow-I_{\mathrm{p}}\right)$ magnetic field orientation. It should be noted that for negative biasing the calculated bias voltage is about a factor 2 smaller than the applied bias potential. This can be explained by the fact that the potential seen by the plasma is different from the imposed bias voltage. Also experimental measurements of floating potential in case of negative biasing yield the same difference in comparison to
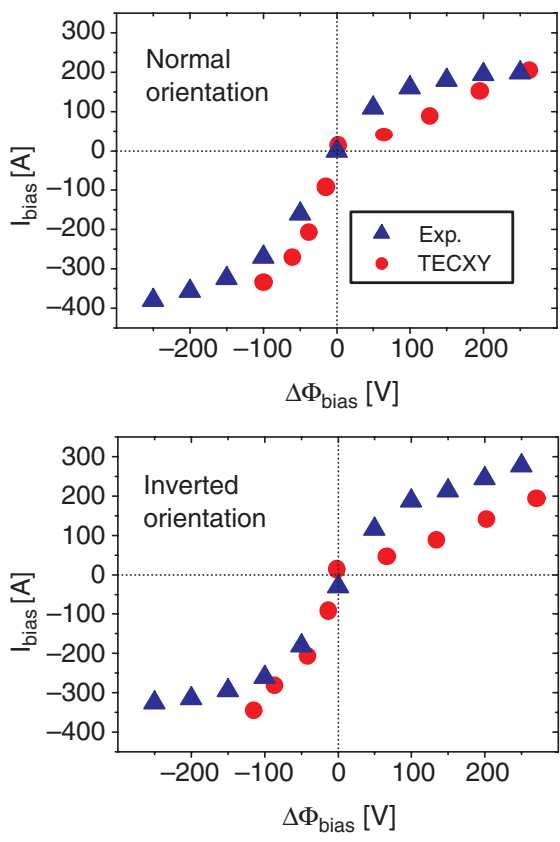

Figure 4. Measured and calculated $I-V$ characteristics for TEXTOR. 

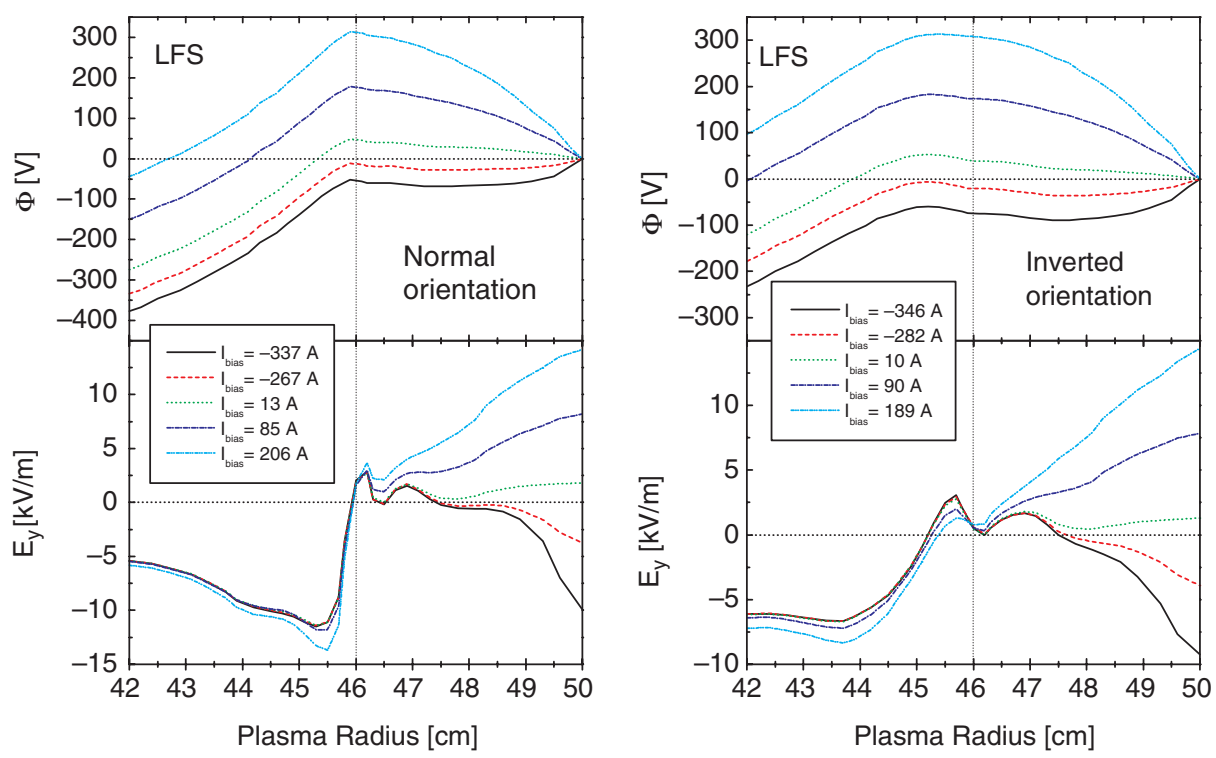

Figure 5. Radial profiles of plasma potential and electric field at outboard midplane (LFS) for both magnetic field orientations (TEXTOR) (inset frames with $I_{\text {bias }}$-values).

the applied voltage [8]. For positive biasing, on the other hand, the bias current of the TECXY model increases somewhat slower with bias voltage than in experiment, especially for inverted orientation. The probable reason is the action of the bumper limiter which collects also poloidal ion currents whereas in the model there are only radial ion currents to the wall. The discrepancy is larger for inverted orientation because in this case the poloidal drifts in the SOL are counterclockwise, such that a larger fraction of particles on their way from outboard midplane (where the maximum particle input occurs) to the ALT-limiter have to pass the bumper limiter. The results of calculations show that biasing can strongly influence the plasma potential in the TEXTOR tokamak edge plasma. However, the corresponding changes to the radial electric field are only limited to the outer part of the SOL (figure 5). The physical mechanisms are somewhat different for both signs of biasing. Whereas for negative biasing the prescribed poloidal bias currents modify the sheath potential at the limiter and thus determine the radial potential profiles in the plasma, for positive biasing the prescribed radial electric fields and poloidal electric drifts lead to poloidal pressure gradients, which in turn produce radial electric currents to be compensated by poloidal currents.

There is only a rather moderate influence of biasing on plasma temperature and density as can be seen in figure 6 . The temperature profiles become very flat for negative biasing. The density profiles, on the other hand, become slightly steeper for negative and slightly flatter for positive biasing. There are similar observations from experiment (figure 7 of [6]). Figure 5 shows the important feature that in case of negative biasing the radial electric field is strongly decreased, whereas for positive biasing it increases. Obviously for some negative bias current the naturally occurring outward directed electric field in the SOL is almost completely compensated.

A similar behaviour of $E_{r}$ is observed also in experiment. This can be seen in figures 7 and 8 where measured (only for negative biasing and inverted orientation) and calculated

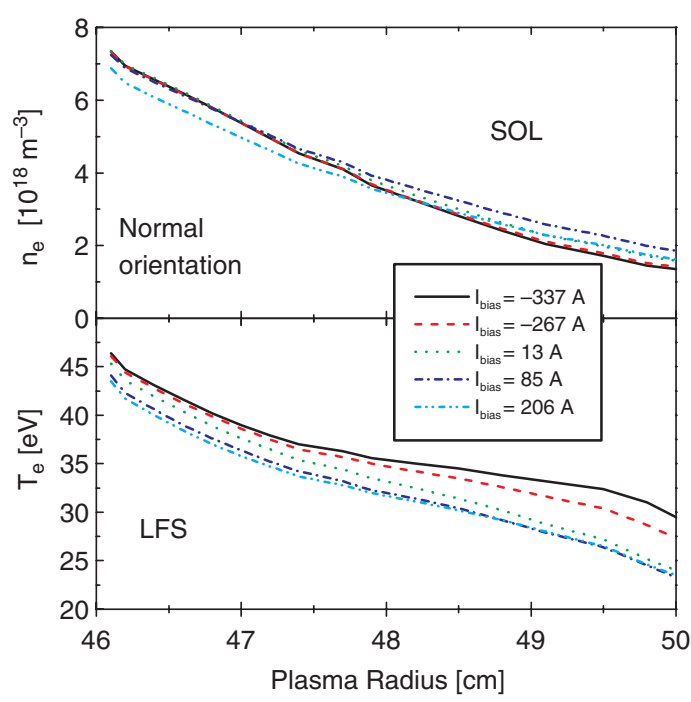

Figure 6. Calculated temperature and density profiles at LFS for normal field orientation.

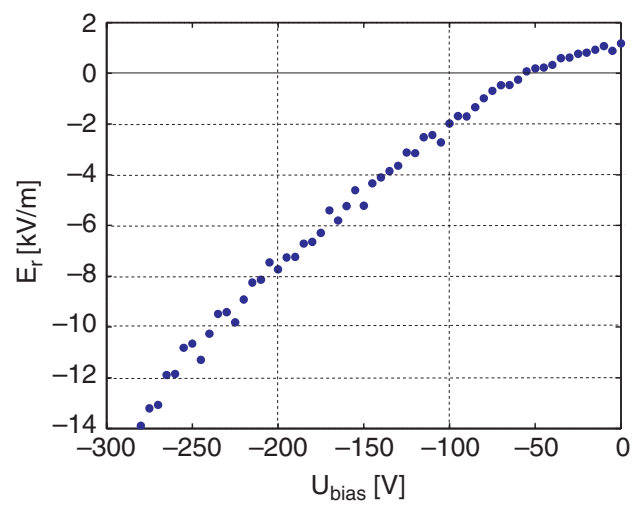

Figure 7. Measured radial electric field at LFS and $y=49.5 \mathrm{~cm}$. 


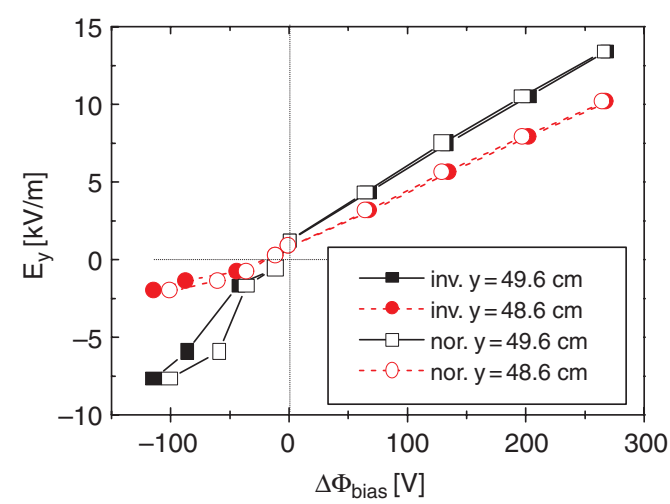

Figure 8. Calculated radial electric field at LFS and two radial positions.

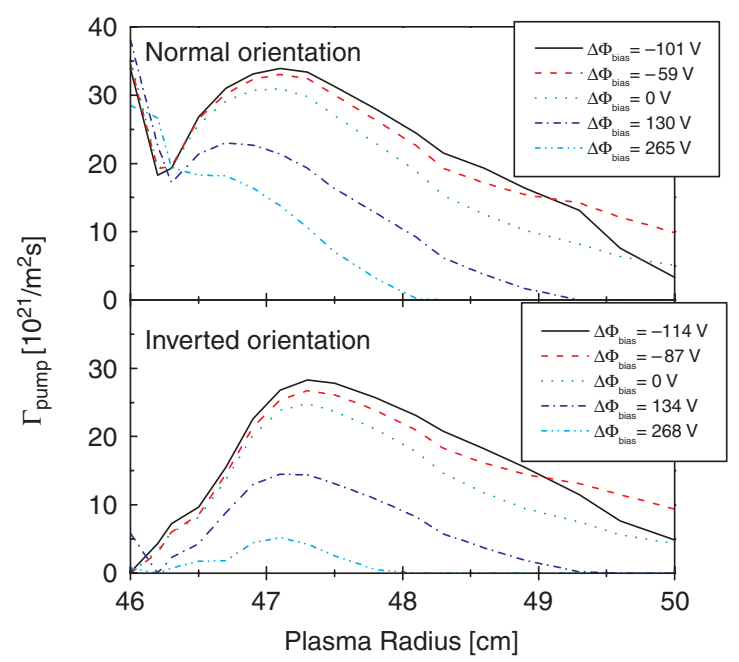

Figure 9. Calculated total plasma flux to the pump for both magnetic field orientations.

values of the radial electric field are shown versus the biasing potential. This feature can have a large influence on plasma flows in the SOL, because the electric drifts (together with the diamagnetic drifts) determine the perpendicular transport in the edge plasma. Keeping in mind that the ALT-limiter scoops are optimally aligned along the total magnetic field for standard TEXTOR operating conditions, the impinging plasma flow can be best pumped away if it is exactly parallel to the $B$ field. For negative biasing the naturally existing perpendicular (electric and pressure gradient) drifts in the SOL can be compensated and hence limiter pumping optimized. In addition the negative limiter helps attracting ions.

For positive biasing, on the contrary, the naturally existing drifts are even enhanced, particles entering the scoop with a large cross-velocity are neutralized already at the side walls of the throats and plug or obstruct further particle exhaust. Additionally positive limiter repels the ions. Consequently experimental exhaust efficiency and pumping capability should be drastically reduced, and the particle flow directed mainly to the wall. The exhaust capability can be estimated in the calculations by an effective pumped flux density defined as $\Gamma_{\text {pump }}=\sum_{\text {es,is }} \operatorname{Max}\left(0,\left|n v_{x}\right|-\left|n v_{\perp}\right|\right)$.

In figure 9 radial profiles of this pumped flux are plotted for different bias voltages. It can be seen that in the case of negative biasing there is an optimal bias voltage for which $\Gamma_{\text {pump }}$ is the largest in the region of the pumping scoops $(y>48 \mathrm{~cm})$.

Similar trends are also observed in the experiments on TEXTOR-94. We can estimate a maximum increase in the pumped flux of about 50\%. This corresponds to an experimental decrease of the measured effective particle confinement time from about $0.9 \mathrm{~s}$ at $0 \mathrm{~V}$ and at $-300 \mathrm{~V}$ to a minimum of about $0.6 \mathrm{~s}$ at $-80 \mathrm{~V}$ (inverted orientation). From figure 9 it can also be seen that positive biasing always reduces the pumping efficiency. The experimental exhaust efficiency for positive biasing, however, is rather unaffected by the bias voltage, which is probably related to the fact that the experimental floating potential profiles are very flat in the scoop region [8]. Hence the experimental radial electric fields in the scoop region are much smaller than in the numerical simulation, which does not take due account of the bumper limiter. The bumper can even induce additional undesired wall fuelling and thus enhance limiter pumping despite reduced pumping efficiency.

Indeed, an increase of about $15-20 \%$ of the collected particle flux was observed for positive biasing and normal orientation in [6]. In any case positive biasing has always the disadvantage for pumping that the limiter repels the ions. We remark that the results for negative biasing and normal orientation in the same [6] cannot be easily compared to the modelling, because they corresponded to special discharge conditions with high confinement (improved mode, even detachment) and strong pumping of the vessel walls such that only few particles were left for limiter pumping, which decreased instead of increased the collected particle flux by the ALT-II limiter.

In figure 10 we compare measured and calculated profiles of parallel and perpendicular Mach numbers at top position. We refer here to normal field orientation and positive biasing (measurements). We should note that experimental values beyond $49 \mathrm{~cm}$ cannot be used since they are in the shadow of obstacles like antenna limiter. The experimental $M_{\text {perp }}$ values are very small and increase with bias potential. The same trend is seen in the calculated profiles. $M_{\|}$with values around -0.15 depends only weakly on radius and bias potential, both in experiment and simulations. In figures 11 and 12 we compare parallel and perpendicular Mach numbers at outboard midplane and for inverted field orientation (in figure 11) and additionally at top position for both field orientations (in figure 12). They are plotted versus the bias potential for fixed radial positions.

We can see that the overall experimental trends are reproduced by the TECXY code results, but of course not all main trends can be well reproduced, in particular the above mentioned reduction of collected particle flux for negative biasing in normal orientation.

\section{Application of the validated model to Tore Supra}

In the case of Tore Supra we used similar boundary conditions as for TEXTOR (see figure 2), but now the computational domain extends poloidally from the limiter ion side at $x_{i}=-2.3 \mathrm{~m}$ to the electron side at $x_{\mathrm{e}}=2.3 \mathrm{~m}$ and radially from $r=66 \mathrm{~cm}$ to $r=80 \mathrm{~cm}$ with the separatrix at $a=72 \mathrm{~cm}$. The width of the transition layer is now $6 \mathrm{~cm}$ (compared to 

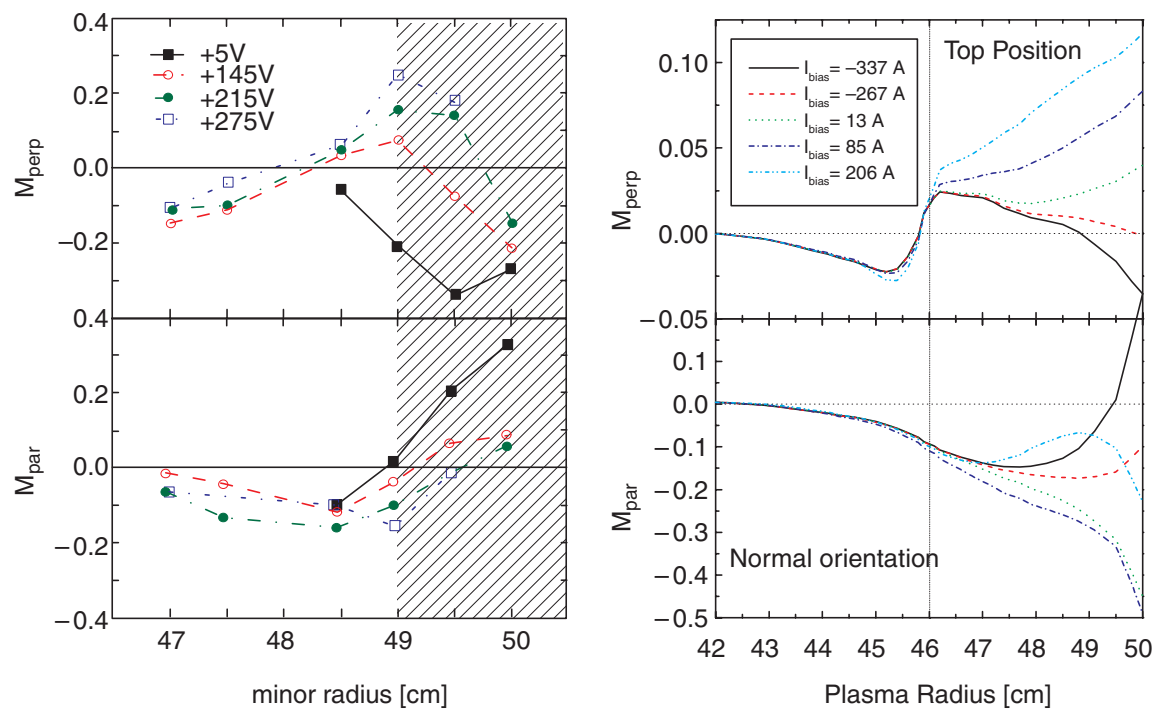

Figure 10. Measured (left) and calculated (right) profiles of parallel $\left(M_{\text {par }}\right)$ and perpendicular $\left(M_{\text {perp }}\right)$ Mach numbers at TOP position for normal field orientation.
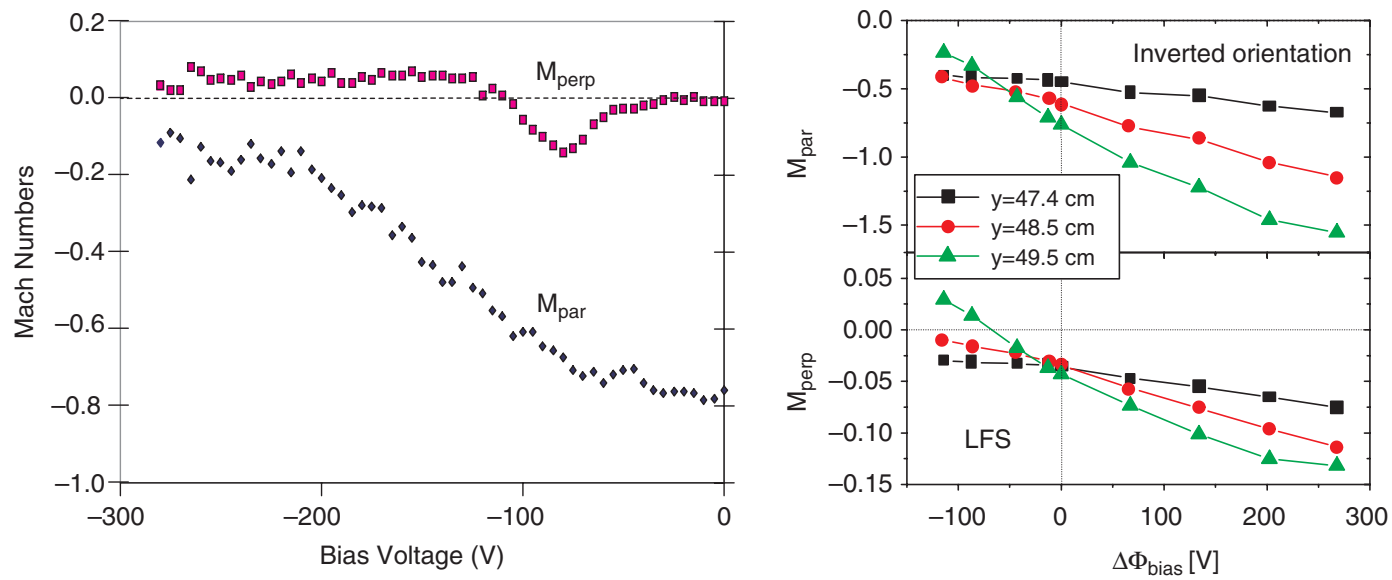

Figure 11. Measured (at $y=49.5 \mathrm{~cm}$, left) and calculated (right) values of $M_{\mathrm{par}}$ and $M_{\text {perp }}$ versus bias potential at LFS for inverted field orientation
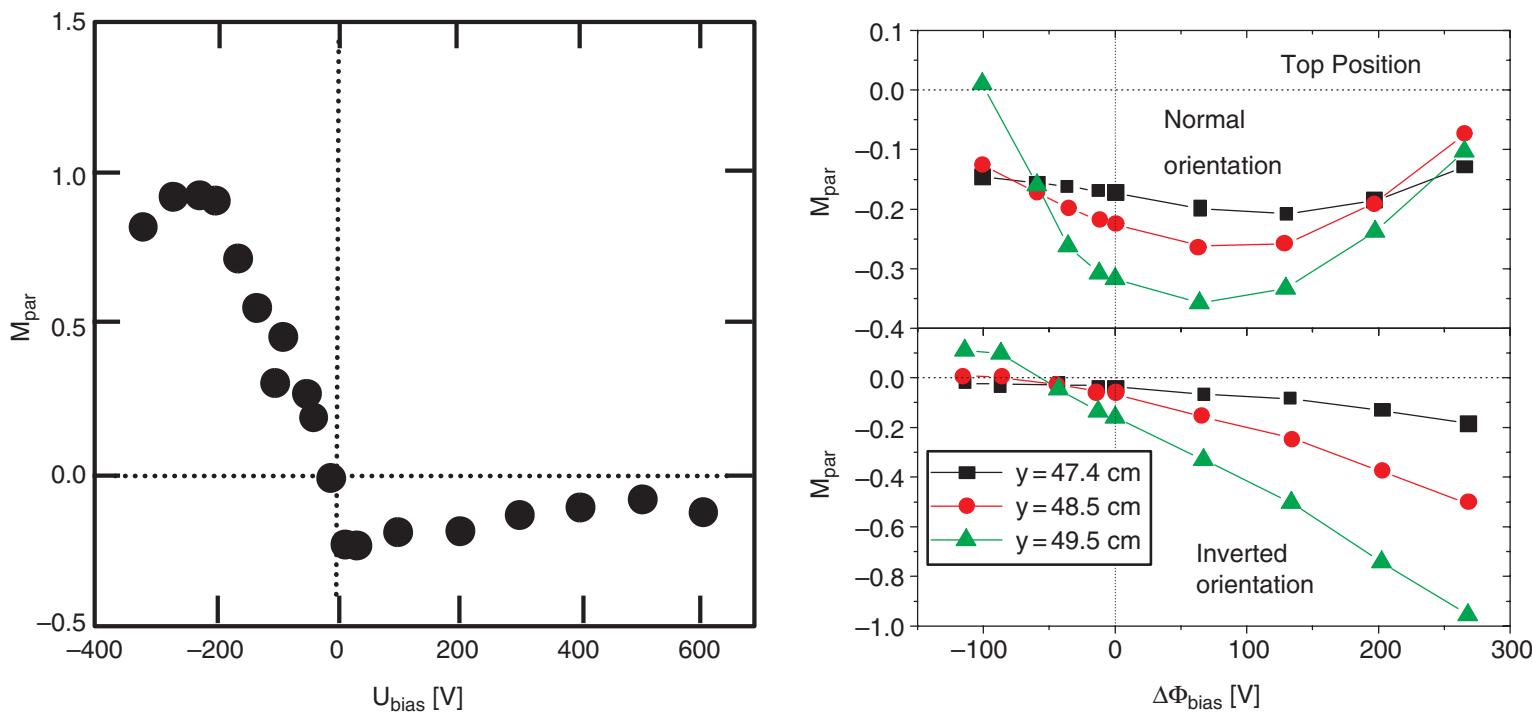

Figure 12. Measured (at $y=49 \mathrm{~cm}$, normal orientation, left) and calculated (for both magnetic field orientations, right) values of $M_{\mathrm{par}}$ versus bias potential at TOP position. 
$4 \mathrm{~cm}$ in TEXTOR) and the width of the SOL is now $8 \mathrm{~cm}$ (twice TEXTOR). The belt limiter TPL is at $\theta=-90^{\circ}$ position, the total magnetic field $B=4.0 \mathrm{~T}$, the plasma current $I_{\mathrm{p}}=1.6 \mathrm{MA}$ and the Shafranov shift $\Delta=13 \mathrm{~cm}$. The anomalous transport coefficients in radial direction are assumed to be the same as for TEXTOR, only the radial viscosity is taken to be twice as large: $\eta_{y}=\frac{2}{3} m_{i} n_{i} D_{y}$. For the present case the input particle flux to the SOL was assumed to be $\Gamma_{\text {inp }}=1.5 \times 10^{22} \mathrm{~s}^{-1}$ and the power input $Q_{\text {inp }}=5 \mathrm{MW}$.

It should be noted that in contrast to TEXTOR the inverted regime of operation is not available in Tore Supra. Also, due to construction limitations, pumping by TPL limiter can be realized only at the $\mathrm{i}$-side (not on both limiter sides as in TEXTOR).

When applying our validated TECXY model to the geometry and parameters of Tore Supra we expected to obtain essentially similar results as for TEXTOR. However, we have to report on quite remarkable differences. First we investigated the $I-V$ characteristics for Tore Supra (figure 13). For negative

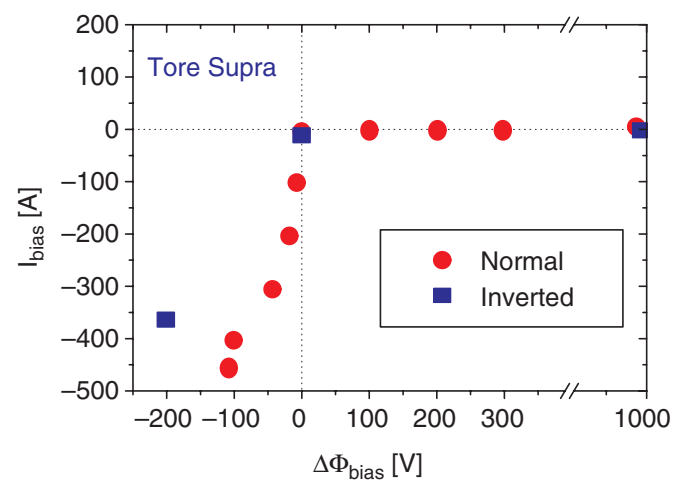

Figure 13. Calculated $I-V$ characteristics for Tore Supra.



biasing we have comparable bias voltages as in the TEXTOR case, but much larger bias currents. This is related to the larger dimensions which allow to achieve increased ion saturation currents to the limiter. There is a steep slope for small negative bias voltages. Already for $\Delta \Phi_{\text {bias }}=-16 \mathrm{~V}$ we have $I_{\text {bias }}=-200 \mathrm{~A}$, which can easily be estimated from the approximate formula

$$
\Delta \Phi_{\text {bias }} \approx \frac{k T_{\mathrm{e}}}{e} \ln \left(1-\frac{I_{\text {bias }}}{I_{\text {sat }}}\right) \approx-\frac{k T_{\mathrm{e}}}{e} \frac{I_{\text {bias }}}{I_{\text {sat }}}
$$

if we assume $k T_{\mathrm{e}} \approx 40 \mathrm{eV}$ and $I_{\mathrm{sat}} \approx-500 \mathrm{~A}$.

We restricted our considerations usually to normal field orientation, because as mentioned in Tore Supra there is no possibility to invert the field orientation. But we point out that for inverted orientation higher SOL temperatures and hence higher sheath voltage drops would occur (the left square in figure 13). Very surprisingly our biasing model does not yield any sensible bias currents for positive biasing, even for $1000 \mathrm{~V}$ bias voltage. This might even be a good reason to really equip Tore Supra with a biasing system.

In order to explain this behaviour we have to note that the relative magnitude and importance of perpendicular drift flows (compared to parallel flows) is much smaller in Tore Supra than in TEXTOR. On the one hand we have larger radial gradient lengths and larger magnetic field, which reduces the perpendicular drift velocities. On the other hand an increased power input and temperature level tends to produce higher sound velocity and poloidal plasma flow towards the limiter.

In figure 14 we can see radial profiles of some plasma parameters at LFS for different bias voltages. It can be noted that by applying our bias model we can produce relatively large changes to the potential and electric field, especially close to the wall, but the corresponding changes to the poloidal velocity are small. Whereas at LFS (remember flux compression by

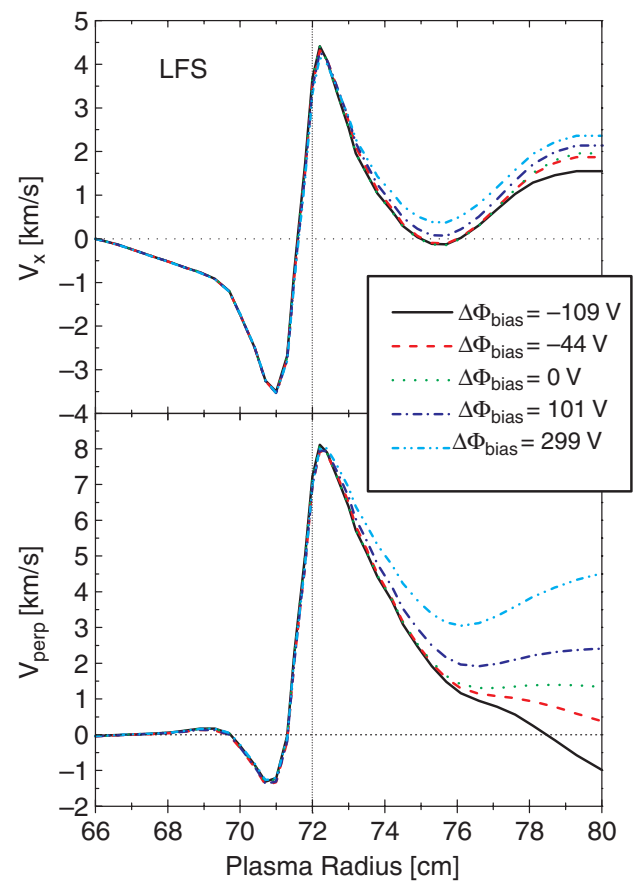

Figure 14. Radial profiles of plasma potential, electric field and velocities $V_{x}, V_{\perp}$ at LFS for Tore Supra (inset frame with $\Delta \Phi_{\text {bias }}{ }^{-v a l u e s) . ~}$ 




Figure 15. Radial profiles of plasma velocities at the TPL i-side (Tore Supra).

Shafranov shift) the velocities $V_{x}$ and $V_{\perp}$ may still be of the same order, at the limiter ion side $V_{\perp}$ is essentially one order of magnitude smaller than $V_{x}$ (see figure 15) and therefore cannot have a sensible effect on the particle flow to the limiter. Thus the poloidal drift flows are also unable to build up a pronounced particle accumulation/depletion at the limiter sides as well as the concomitant poloidal pressure gradients, which then cannot lead to radial diamagnetic drifts and bias currents in the case of positive biasing. Even significant changes to the electric field in the outer region of the tokamak SOL cannot produce corresponding changes in the poloidal profiles of plasma pressure and consequently the resulting radial currents are negligible.

The effect of biasing on plasma density and temperature profiles is equally tiny and almost invisible (figure 16). The same is true for the poloidal and projected parallel particle fluxes to the limiter i-side (figure 17). Biasing has obviously no sensible influence in this case. We did not show the electron side because, as mentioned, Tore Supra has no pumping throats at the e-side, but the situation at the e-side is very similar.

In principle, for moderate negative biasing we have again the same effect as in TEXTOR that the naturally existing drifts in the SOL may be compensated and even inverted, which optimizes the pumping exhaust efficiency for scoop throats aligned parallel to the total magnetic field. So we again calculated the estimated $\Gamma_{\text {pump }}=\operatorname{Max}\left(0,\left|n v_{x}\right|-\left|n v_{\perp}\right|\right)$, but only for the $\mathrm{i}$-side, and indeed for small values of the negative biasing voltage there is an increase of $\Gamma_{\text {pump }}$, but smaller than $5 \%$ and hence negligible (figure 18). Even the reduction of $\Gamma_{\text {pump }}$ for positive biasing is very much smaller than for TEXTOR. This means that biasing is probably less effective in Tore Supra than in TEXTOR for the purpose of improving

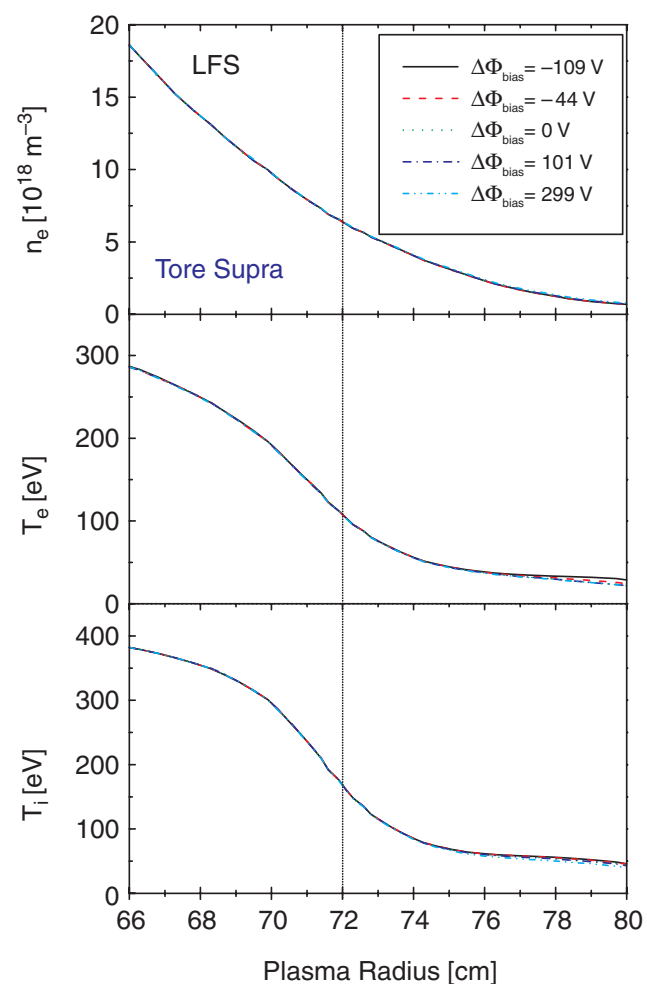

Figure 16. Radial density and temperature profiles at LFS for Tore Supra.

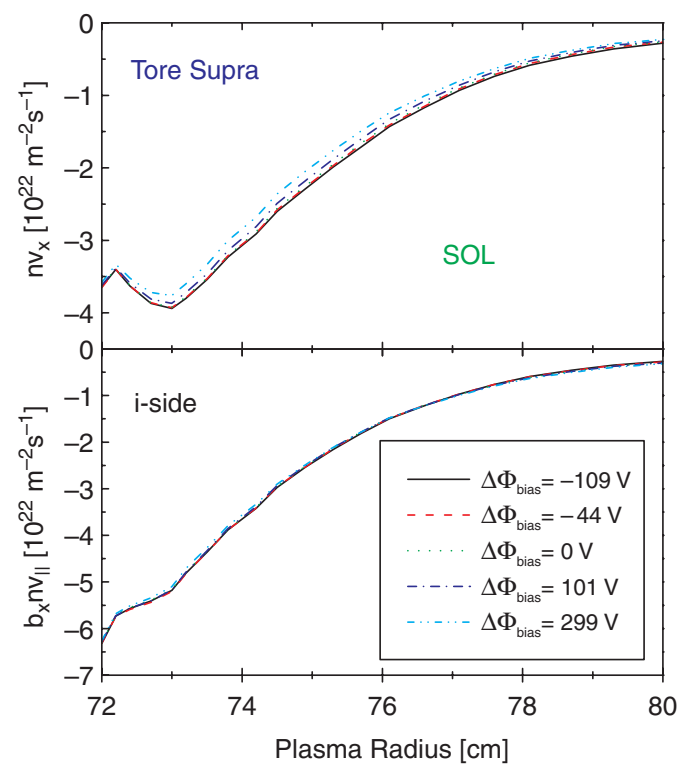

Figure 17. Plasma particle fluxes to the TPL i-side.

the pumping performance of TPL, at least in the considered range of parameters. On the other hand the mere fact that the model predicts such a difference between TEXTOR and Tore Supra absolutely requires that the experiment be performed as a validation. Also the pumping or refuelling properties of the vessel wall (effect of recycled neutrals) and the action of antenna protection limiters may strongly modify the particle collection and pumping efficiency of TPL, which must be further investigated experimentally. 


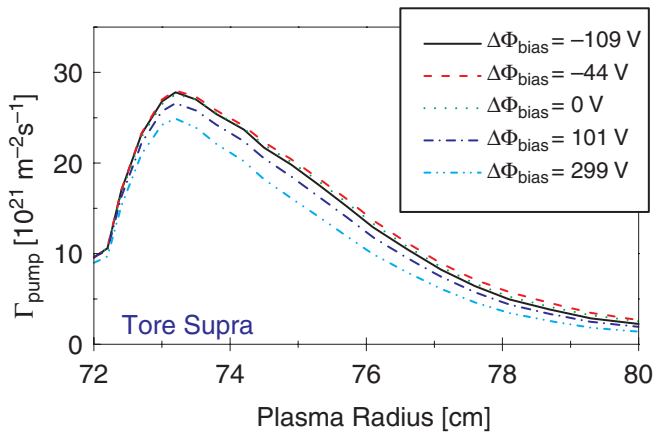

Figure 18. Total particle flux density to the pump for Tore Supra.

\section{Conclusions}

Keeping in mind that the belt limiter scoops (in ALT-II as well as in TPL) are optimally aligned along the total magnetic field for standard tokamak operating conditions, the impinging plasma flow can be best pumped away if it is exactly parallel to the $B$ field. For negative biasing the naturally existing perpendicular drifts in the SOL can be compensated and hence limiter pumping optimized. For positive biasing, on the contrary, the naturally existing drifts are even enhanced, particles enter the scoop with a large cross-field velocity, are neutralized already at the side walls of the throats and plug or obstruct further particle exhaust. Consequently experimental exhaust efficiency and pumping capability should be drastically reduced. These conclusions hold quite similarly also for inverted field orientation and cannot be affected by varying discharge conditions (improved or low confinement mode).

In TEXTOR the experimental $I-V$ characteristics for either biasing of ALT-II, the radial profiles of electric fields, Mach numbers and other plasma parameters are quite well reproduced by the code modelling. The improvement of the limiter pumping exhaust efficiency amounts up to $50 \%$. The application of the validated model to the analogous biasing problem in Tore Supra leads to very surprising predictions. Since the relative magnitude and importance of the perpendicular drift flows (compared to parallel flows) results to be much smaller in Tore Supra than in TEXTOR, the bias-induced changes of plasma parameters and particle flows to the limiter pumping scoops are drastically reduced. The possible improvement of limiter performance for negative biasing is smaller than $5 \%$ and hence negligible. Nevertheless biasing may be useful, so we recommend to try biasing as a possible means for increasing the pumping exhaust in Tore Supra, also because it would be scientifically extremely interesting to validate or disprove the theoretical model predictions.

\section{References}

[1] Zagórski R., Gerhauser H. and Claaßen H.A. 1999 J. Nucl. Mater. 266-269 1261

[2] Reiser D. et al 1999 26th EPS Conf. on CFPP (Maastricht, 1999) ECA vol 23J, p 697

[3] Gerhauser H. et al 2000 Contrib. Plasma Phys. 40309

[4] Gerhauser H. et al 2001 J. Nucl. Mater. 290-293 609

[5] Zagórski R. et al 2000 Contrib. Plasma Phys. 40405

[6] Loarer T. et al 2000 Czech. J. Phys. 501441

[7] Weynants R.R. and vanOost G. 1993 Plasma Phys. Control. Fusion B 35177

[8] Mank G. et al 2000 Proc. 10th Int. Congr. on Plasma Physics (Québec City, Canada) vol 3, p 760

[9] Doerner R.P. et al 1994 Nucl. Fusion 34975

[10] Gerhauser H. et al 2001 Czech. J. Phys. $\mathbf{5 1} 1033$

[11] Braginskij S.I. 1965 Rev. Plasma Phys. 1205

[12] Claaßen H.A. et al 1991 Report of KFA Jülich 2423

[13] Tokar M.Z. 2000 Contrib. Plasma Phys. 40443

[14] Zagórski R., Gerhauser H. and Claaßen H.A. 1999 J. Techn. Phys. 4099

[15] Zagórski R., Gerhauser H. and Claaßen H.A. 1998 Contrib. Plasma Phys. 3861

[16] Lehnen M. et al 2001 J. Nucl. Mater. 290-293 663

[17] Connor J.W. et al 1999 Nucl. Fusion 39169

[18] Zagórski R. et al 2000 Report of KFA Jülich $\mathbf{3 8 2 9}$

[19] Stangeby P.C. and Chankin A.V. 1995 Phys. Plasmas 2707

[20] Claaßen H.A. and Gerhauser H. 1996 Contrib. Plasma Phys. 36361

[21] Hutchinson I.H. 1996 Phys. Plasmas 36 